\title{
Role of Combined Blood Neutrophil- Lymphocyte Ratio and C-reactive Protein in Diagnosis of Spontaneous Bacterial Peritonitis
}

\author{
S.A.Awad, E.S.Ahmed and E.E.Mohamed
}

Hepatology, Gastroenterology and Infectious Disease Dept., Faculty of Medicine, Benha Univ., Benha, Egypt

E-Mail:ahmed2356@gmail.com

\begin{abstract}
Ascites is the most common complication of cirrhosis, constitutes the first manifestation of disease in the majority of patients and is the most common cause of hospitalization in the cirrhotic patient. Bacterial infection of ascites in the cirrhotic patient's increases mortality up to four fold, The aim of the study is to assess the clinical usefulness of C - reactive protein and Neutrophil- Lymphocyte Ratio in diagnosis of ascetic fluid infection, this is cross-sectional study that was conducted in the Department of Hepatology, Gastroenterology and Infectious diseases-Banha university hospital .The study was carried out on 140 Patients with chronic liver disease, divided in to two groups according to presence or absence of SBP, the results revealed that there was statistical significant difference between two studied groups regarding WBCs, neutrophils, hemoglobin and CRP, Basal CRP level is generally higher in patients with SBP than without SBP. During bacterial infections CRP level rises and we concluded that Combined NLR and CRP could be used as a novel, simple, lowcost, non-invasive test for SBP diagnosis.
\end{abstract}

Keywords: C-reactive protein, Neutrophil, Spontaneous bacterial peritonitis, Chronic liver.

\section{Introduction}

Ascites is the most common complication of cirrhosis, conistitutes the first manifestation of disease in the majority of patients and is the most common cause of hospitalisation in the cirrhotic patient. Bacterial infection of ascites in the cirrhotic patients increase mortality up to four fold [1].

Spontaneousbacterial peritonitis $[\mathrm{SBP}]$ is a serious common form of infection in cirrhotic ascitic patients Its prevalence among the hospitalisedgroup of those patients varies from 10 to $30 \%$ [2].

Normally the ascietic fluid is transparent and amber yellow in color and the polymorphonuclear neutrophil [PMN] concentration in ascitic fluid is less than 250 cells per litre [3].

SBP is a consequence of qualitative and quantitative changes in microbiota of the gut, bacterial translocation and increased permeability of the intestine]. SBP has been defined as an ascitic fluid infection associated with positive bacterial culture and ascitic fluid polymorphonuclear [PMN] cell count more than 250/mm3 [4].

As signs and symptoms of SBP are non-specific and the presentation varies according to the stage of the disease, diagnosis depends mainly on the laboratory and microbiological tests. Paracentesis and laboratory examination of the ascitic fluid is considered the gold standard test to confirm the diagnosis of SBP in cirrhotic patients [5].

The diagnostic paracentesis risks include visceral perforation, haemoperitoneum, peritonitis, local infection at the site of paracentesis, abdominal wall haematoma and persistant leak, which is the most common complication [6].

C-reactive protien [CRP] is increased in the blood when there is an inflammatory lesion in the living body. Serum CRP is thought to be synthesised only in hepatocytes by the induction of cytokines such as interleukin-1, interleukin-6 and tumour necrosis factor produced by macrophages at the time of bacterial infection [7].

The blood neutrophil lymphocyte ratio [NLR] is an important index for the balance of the inflammatory and immune systems, reflecting systemic inflammation responses [8]. Platelet indices, CRP and total white blood cell counts are reported to predict ascitic fluid infection [9].

The aim of the study was to assess the clinical usefulness of C - reactive protein and NeutrophilLymphocyte Ratio in diagnosis of ascitic fluid infection.

\section{Patient and method}

This was as a cross-sectional study conducted in the Department of Hepatology, Gastroenterology and Infectious diseases-Benha University Hospital between Mars 2019 \&December 2019.The study had included 140 Patients with chronic liver disease.

All the investigation done in clinical and chemical pathology department Benha University Hospital.

Patients were divided into two groups:

Group [1]: 70 patients with liver cirrhosis and ascites without evidence of Spontaneous Bacterial Peritonitis [SBP].

Group [2]: 70 patients with liver cirrhosis, ascites and SBP.

All patients had subjected to full history taking, complete Clinical examination and Laboratory Investigations including:

a. Complete blood count with calculation of Neutrophil/Lymphocyte ratio.

b. Random blood sugar.

c. Liver profile tests:

- Serum albumin.

- Serum aspartate transaminase [AST].

- Serum alanin transaminase [ALT].

- Bilirubin [total and direct]. 
- INR, prothrombin time and concentration.

d. Kidney function test: Serum creatinine.

e. Ascitic fluid Analysis[diagnostic paracentesis] for:

1. Biochemical analysis of glucose, albumin and total protein.

2. Cytological analysis of Total Leucocytic Count.

This data was taken from patient file

f. Quantitative C. reactive. protein measurement

\section{Sampling}

Five milliliters of venous blood were drawn

Two milliliters were put in EDITA -vacutainer, tube for determination of CBC and of Neutrophil/Lymphocyte ratio the rest of the venous the venous sample was put in plain tube and let it to coagulate for about 30min then centrifugated at $1200 \mathrm{rpm}$, the serum separated and used for determination of CRP.

\section{Inclusion criteria}

1. Liver cirrhosis diagnosed on clinical, laboratory and radiological findings supported by liver biopsy whenever possible.

2. Patients aged more than 18 years old.

3. Spontaneous bacterial peritonitis diagnosed by: a-Clinical picture [fever, abdominal, pain, vomiting and confusion].

b-Ascetic fluid examination with PMN >250/ml.

\section{Exclusion Criteria}

Patients with the following conditions were excluded:

1- Non cirrhotic Ascites.

2- Immunocompromised patients.

3- Patients with secondary bacterial peritonitis.

4- Patients who received antibiotics prior to hospitalization or under prophylactic antibiotics for spontaneous bacterial peritonitis.

\section{Results}

The present study shows that for the without SBP group there were 66 [94.3\%] males and 4 [5.7\%] females, the mean age was $61.50 \pm 6.22$ with range of [52-73]. For the with SBP group there were 70 [100\%] males and $0[0 \%]$ females, the mean age was $58.60 \pm$ 6.78 with range of [50-74].

There was significant difference between the studied groups as regard Age. There was high significant difference between the studied groups as regard abdominal pain, Loss of weight and Fever and significant difference as regard Bilharziasis and Bleeding with increase in SBPgroup..

The present study shows that for the without SBP group there 54 [77.1\%] with no history of operation and 16 [22.9\%] with history; among those with history there were 4 [25\%] with C.S, 4 [25\%] with Hernia, 4 [25\%] with Piles and 4 [25\%] with Appendicictomy.

For the with SBP group there 61 [88.4\%] with no history of operation and 8 [11.6\%] with history; among those with history there were 4 [50\%] with Inguinal hernia.
There was high significant difference between the studied groups as regard History of operation. There was high significant difference between the studied groups as regard Pallor, Ascites and Flapping tremors.

This table shows that for the without SBP group the mean RBS was $148.6 \pm 59.18$ with range of [66.8-283], the mean HGB was $9.87 \pm 1.73$ with range of [5.7-13.7], the mean WBCs was $7.93 \pm 5.25$ with range of $[2.8$ 24.3], the mean Neutrophils was $6.15 \pm 4.54$ with range of [1.9-20.2], the mean Lymphocytes was $1.21 \pm 0.61$ with range of [0.4-2.6] and the mean PLT was $139.8 \pm$ 94.67with range of [43-307].

For the with SBP group the mean RBS was $143.9 \pm$ 55.10 with range of [48-292], the mean HGB was $9.05 \pm$ 1.96 with range of [6.7-14.7], the mean WBCs was 13.52 \pm 7.54 with range of [3.9-34.3], the mean Neutrophils was $10.86 \pm 7.08$ with range of [3.4-31.9], the mean Lymphocytes was $1.20 \pm 0.68$ with range of [0.1-2.5] and the mean PLT was $151.6 \pm 148.7$ with range of [55737].

There was high significant difference between the studied groups as regard WBCs and Neutrophils and significant difference as regard HGB.

The present study shows that for the without SBP group the mean NLR was $5.65 \pm 3.80$ with range of [1.76-17.75]. For the with SBP group the mean NLR was $26.34 \pm 72.67$ with range of [3.52-319]. There was high significant difference between the studied groups as regard NLR.

The present study shows that for the without SBP group the mean $\mathrm{Cr}$ was $2.29 \pm 1.33$ with range of [0.374.59], the mean INR was $1.59 \pm 0.41$ with range of [12.48 ], the mean PT was $17.71 \pm 3.07$ with range of [1222.5], the mean CRP was $51.28 \pm 47.46$ with range of [1.3-169].

for the with SBP group the mean $\mathrm{Cr}$ was $1.55 \pm 0.86$ with range of [0.57-4.76], the mean INR was $1.86 \pm 0.55$ with range of [1-3.2], the mean PT was $19.42 \pm 4.27$ with range of [13.8-32.2], the mean CRP was $78.52 \pm 42.0$ with range of [9.9-163]. There was high significant difference between the studied groups as regard CRP and significant difference as regard Cr, INR and PT.

The present study shows that for the without SBP group the mean ALT was $48.69 \pm 32.37$ with range of [8103.5], the mean AST was $84.29 \pm 62.42$ with range of [17.4-234.8], the mean Serum albumin was $2.70 \pm 0.39$ with range of [1.69-3.3], the mean Total bilirubin was $4.09 \pm 3.86$ with range of [0.41-12.98], the mean Direct bilirubin was $2.66 \pm 2.77$ with range of [0.23-9.51].

For the with SBP group the mean ALT was $55.47 \pm$ 62.79 with range of [10.6-320], the mean AST was 68.85 \pm 63.31 with range of [21.2-323.4], the mean Serum albumin was $2.39 \pm 0.66$ with range of [1.1-3.89], the mean Total bilirubin was $3.52 \pm 2.27$ with range of [1$7.85]$, the mean Direct bilirubin was $2.13 \pm 1.40$ with range of [0.48-5.17].

There was high significant difference between the studied groups as regard Serum albumin.

This table shows that for the without SBP group the mean TLC was $290.8 \pm 99.09$ with range [100-500], the 
mean PMN was $127.0 \pm 47.30$ with range [60-200], the mean Albumin was $0.95 \pm 0.48$ with range [0.5-2.05] and the mean Glucose was $133.2 \pm 55.21$ with range [80.9311].

For the with SBP group the mean TLC was $1734.3 \pm$ 1270.3 with range [300-4700], the mean PMN was $1003.6 \pm 1076.2$ with range [250-4400], the mean Albumin was $0.75 \pm 0.71$ with range [0.11-2.3] and the mean Glucose was $139.7 \pm 79.60$ with range [63.5389.9].

There was high significant difference between the studied groups as regard TLC, PMN and Albumin.

There was high significant difference between the studied groups as regard Liver size and Ascites with increase in SBPgroup..
The present study shows that for the without SBP group For NLR there was strong positive correlation with WBCs, Neutrophils and TLC and negative a correlation with Lymphocytes, Cr, Serum albumin and Albumin.

Using roc curve, it was shown that NLR level can be used to discriminate between patients and controls at a cutoff level of > 3.5, with $100 \%$ sensitivity, $42.86 \%$ specificity, 63.6\% PPV and 100\% NPV.

CRP level also can be used to discriminate between patients and controls at a cutoff level of $>43.3$, with $77.14 \%$ sensitivity, $62.86 \%$ specificity, $62.86 \%$ PPV and $73.3 \%$ NPV.

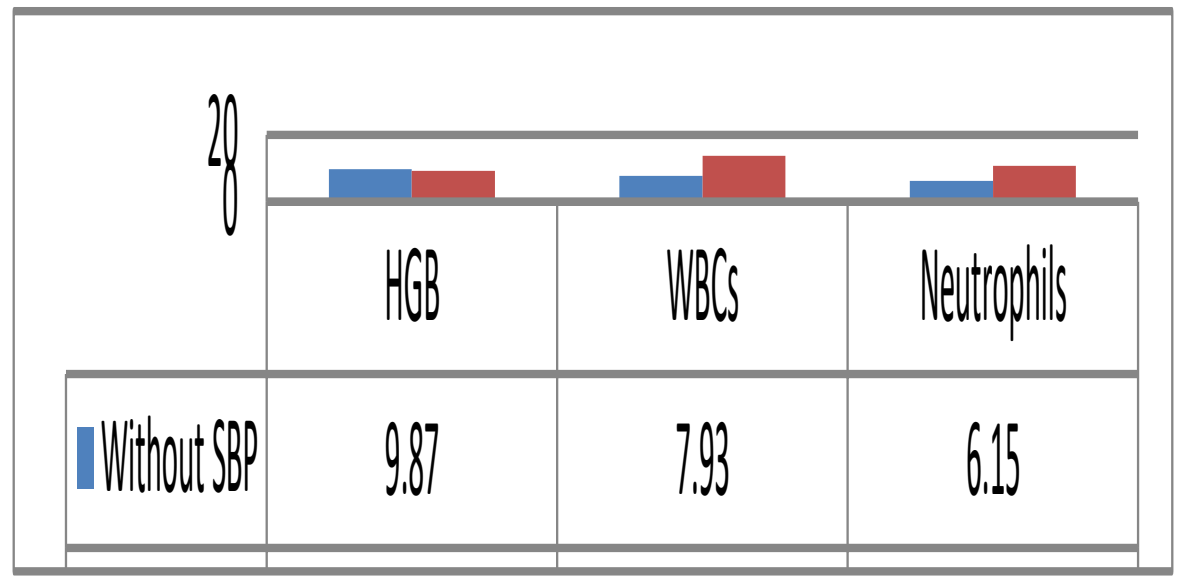

Fig (1) comparison between the studied groups as regard CBC.

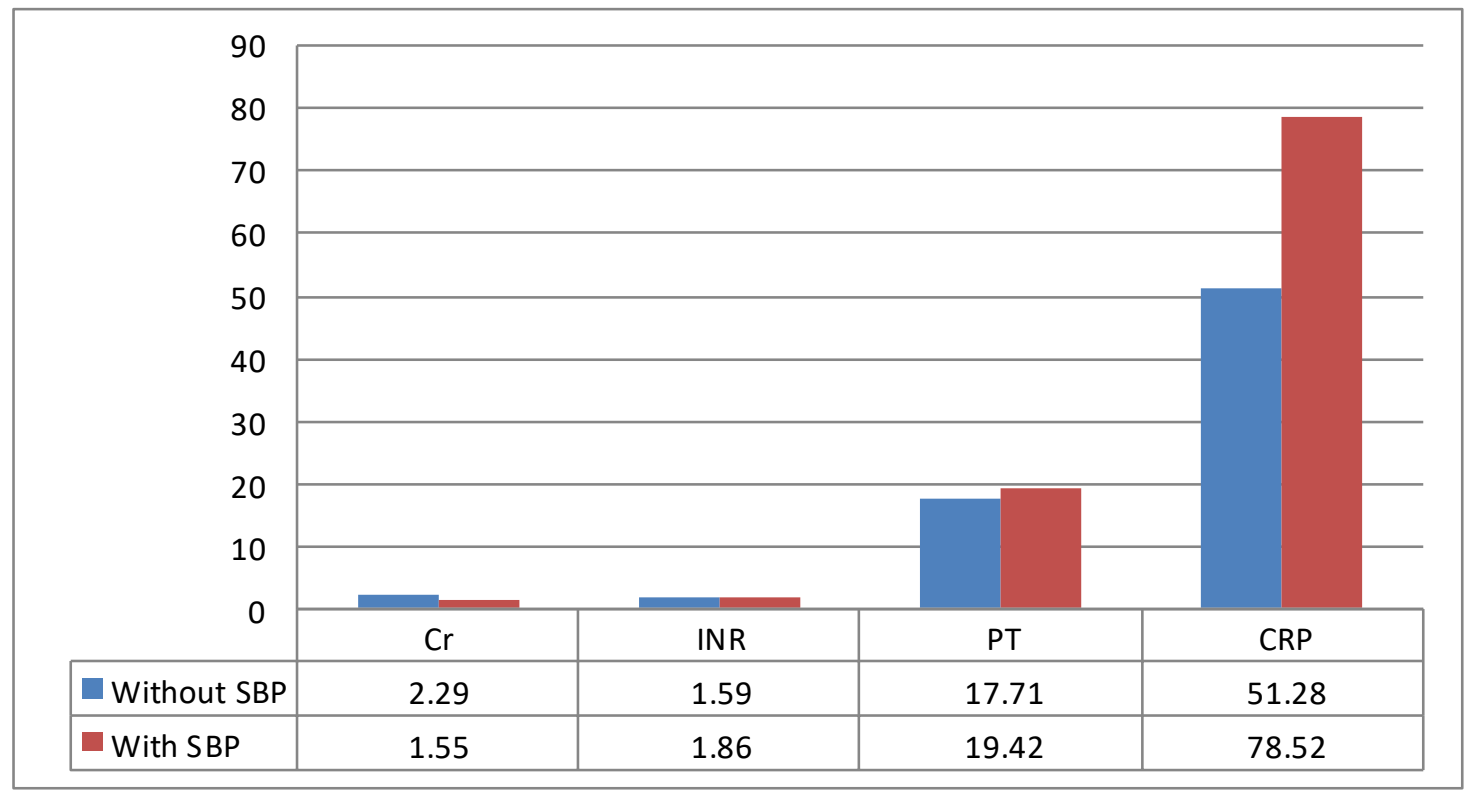

Fig (2) comparison between the studied groups as regard different parameters 


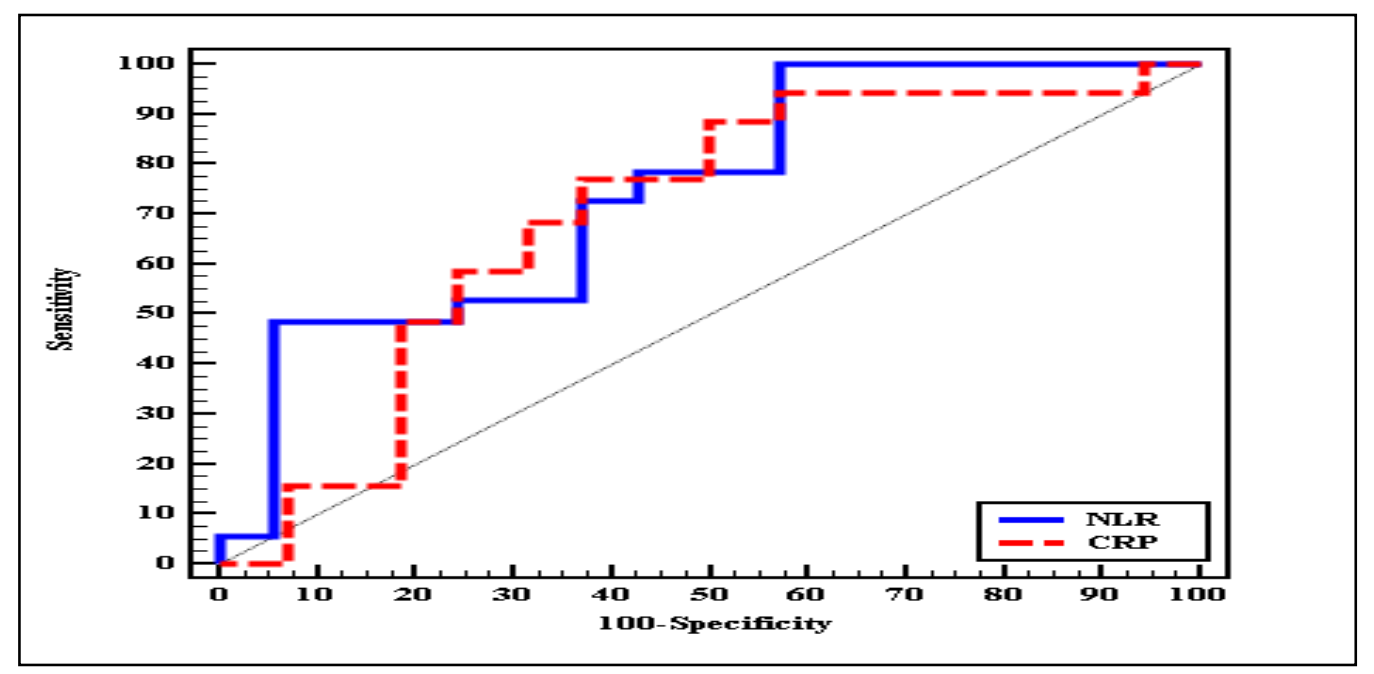

Fig (3) ROC curve for NLR and CRP to predict With SBP [n = 70] from Without SBP [n = 70].

\section{Discussion}

Spontaneous bacterial peritonitis $[\mathrm{SBP}]$ is a serious common form of infection in cirrhotic ascitic patients. Its prevalence among the hospitalised group of those patients varies from $10 \%-30 \%$. About half of them have SBP at admission; the other half develops SBP during hospitalisation [3].

SBP is a consequence of qualitative and quantitative changes in microbiota of the gut, bacterial translocation and increased permeability of the intestine. Also, the impairments of the immune system observed in advanced cirrhotic patients play an important role [10].

Paracentesis and laboratory examination of the ascitic fluid is considered the gold standard test to confirm or rule out the diagnosis of SBP in cirrhotic patients [5].

The diagnostic paracentesis risks include, visceral perforation, haemoperitoneum, peritonitis, local infection at the site of paracentesis, abdominal wall haematoma and persistent leak, which is the most common complication [6].

$\mathrm{C}$ reactive protein [CRP] is increased in the blood when there is an inflammatory lesion or histologically necrotic lesion in the living body. Serum CRP is thought to be synthesised only in hepatocytes by the induction of cytokines such as interleukin- 1, interleukin-6 and tumor necrosis factor produced by macrophages at the time of bacterial infection [11].

The blood neutrophil lymphocyte ratio [NLR] is an important index for the balance of the inflammatory and immune systems, reflecting systemic inflammation responses. Platelet indices, CRP, and total white blood cell counts are reported to predict ascites fluid infection [9].

The main objective of this study was to assess the clinical usefulness of $\mathrm{C}$ - reactive protein and Neutrophil- Lymphocyte Ratio in diagnosis of ascitic fluid infection.

A cross-sectional study was conducted in the Department of Hepatology, Gastroenterology and Infectious diseases-Banha university hospital between
Mars 2019 and December2019. The study was carried out on 140 Patients with chronic liver disease. The patients were divided into two groups: Group [1]: including 70 patients with liver cirrhosis and ascites without evidence of SBP. Group [2]: including 70 patients with liver cirrhosis, ascites and SBP

The two studied groups were compared as regard the demographic data showing no significant difference between the studied groups except for the age [P=0.009].

Contrary to the study of [9] [12] who reported that there was no statistically significant difference between infected and non- infected groups regarding age. These findings largely exclude demographic factors as confounding factors.

As regard the past history including, abdominal pain, Loss of weight, Fever, Bilharziasis, encephalopathy, Bleeding. ] A significant difference was detected between the groups only as regards abdominal pain, fever, loss of weight and Bilharziasis. These parameters were more likely associated with SBP group.

These results are supported by [9] [13] who a statistically significant difference between studied groups regarding abdominal pain,fever and History of operation.

Our results showed that, the studied groups were compared as regards history of operations There was a highly significant difference between the studied groups, where the group without SBP had higher history of operations.

Our results showed that there was significant difference between the studied groups as regards History of Drug intake [antibilharizial, antihypertensive, propranolol and carbimazole], where the group without SBP had more history of drugsClearly, the cases with SBP had more advanced liver disease and needed more treatment

Our results showed that there was a highly significant difference between the studied groups as regards Pallor, Ascites and Flapping tremors.

Our results showed that the studied groups were compared regarding $\mathrm{WBC}, \mathrm{RBC}, \mathrm{HB}$, Neutrophils, 
lymphocytes and platelet. There was a highly significant difference between the studied groups as regards WBCs and Neutrophils and a significant difference as regards HGB. The neutrophils and WBCs count were higher in [SBP] group than in non-SBP group, while HB concentration was higher in non-SBP group.

Our results showed that there was a highly significant difference between the studied groups as regard NLR, where NLR was higher in SBP group than in non-SBP group.

Our results are in agreement with [14] [13] whofound that NLR in infection group were significantly higher than in non-infection group $[\mathrm{P}<0.001]$.

In the general population, $\mathrm{C}$-reactive protein [CRP] level increases in the presence of acute or chronic inflammation and infections. In patients with cirrhosis, the basal level is higher than in patients without cirrhosis, due to chronic hepatic and other inflammation, but when infection occurs the more severe the underlying liver dysfunction, the lower the increase in CRP. Therefore, the predictive power of CRP for infection and prognosis is weak in patients with decompensated/advanced cirrhosis and in the intensive care setting. However, higher CRP and also persistently elevated CRP levels can help identify patients with a higher short-term risk of mortality [15].

Our results showed that there was a highly significant difference between the studied groups as regards CRP, $\mathrm{Cr}$, INR and PT $[\mathrm{P}=0.001,0.002,0.002$ and 0.007 respectively].

Biomarkers have been gaining recognition as an important tool in the diagnosis of bacterial infection. An ideal biomarker would enable physicians to rapidly and reliably establish the diagnosis of a bacterial infection in different patients. CRP, an acute-phase protein produced by the liver in response to infection, is potentially such a biomarker. CRP in healthy individuals is less than 0.5 $\mathrm{mg} / \mathrm{l}$, and when these levels are elevated, it can be useful in establishing the etiology of some infections [16].

CRP is an acute-phase reactant that binds to different substrates. It activates the complements, plays a role in cytokine secretion, and increases the phagocytosis of leukocytes. CRP has been reported to be a reliable predictor of SBP and an index of therapeutic effectiveness in adults [17].

CRP is a more sensitive and accurate reflection of the acute-phase response than the erythrocyte sedimentation rate [ESR]. ESR may be normal and CRP may be elevated. CRP returns to normal more quickly than ESR in response to therapy [18].

A.Tsiakalos [19] analysed the diagnostic value of several acute-phase proteins [CRP, ferritin, $\beta 2$ microglobulin and others] as indicators of bacterial infection in 88 patients with cirrhosis and found that CRP was the best test for detecting bacterial infection [AUROC 0.91]. they proposed a cut-off value of 55.8 $\mathrm{mg} / \mathrm{L}$, which showed high sensitivity [79\%] and specificity [96\%], with the best diagnostic accuracy [92\%].
N.Mousa [13] reported that CRP was significantly higher compared to non SBP, but there was no significant difference in patients with culture positive SBP versus culture negative SBP. A previous study conducted by [20] concluded that the level of CRP alone is not an accurate marker for the diagnosis of SBP.

G.Pieri [15] found that the basic level of CRP in patients with cirrhosis is higher than in patients without cirrhosis, but when infection occurs the more serious the potential liver dysfunction, the lower the increase in CRP. [21] concluded that the power of CRP for infection prediction is weak in patients with advanced cirrhosis.

Therefore, CRP has a weak predictive power for infection in patients with decompensated cirrhosis, as confirmed also by Le [22] who showed that CRP correlated weakly with IL-6 levels in infected patients [where IL-6 was >200 pg/ mL], indicating a defective acute phase response in cirrhosis.

Moreover, in a cohort of 148 patients with decompensated cirrhosis admitted to a single Hepatology unit, [23] found baseline CRP levels to be higher in patients with infection [46 mg/L vs $27 \mathrm{mg} / \mathrm{L}]$ than in patients without infection.

In the work of [9], there was a significant increase in CRP in the SBP group versus the non-SBP group [P < $0.001]$, with a significant positive correlation being observed between serum MPV and CRP [ $\mathrm{r}=0.68, \mathrm{P}<$ $0.001]$. It was reported that at a cutoff value of 10.5 $\mathrm{mg} / \mathrm{dl}$, CRP had $77.4 \%$ specificity and $85.7 \%$ sensitivity for detecting SBP.

M. Preto-Zamperlini [17] reported that the SBP group showed significantly elevated CRP levels, leading to the conclusion that CRP was an independent variable in the prediction of SBP. In a study by [24] it was found that CRP was increased in the serum and ascitic fluid of SBP patients.Lee et al [25] suggested combining NLR and CRP as a novel noninvasive test for SBP diagnosis.

studied groups were compared regarding liver function tests [albumin, total and direct bilirubin, ALT and AST]. There was a highly significant difference between the studied groups only as regards serum albumin.

Our results showed that the two groups were compared as regards ascetic fluid lab [TLC, PMN, albumin and glucose]. There was a highly significant difference between the studied groups as regards TLC, PMN and Albumin.

.Regarding abdominal ultrasonography, there was a highly significant difference between the studied groups as regards Liver size and Ascites with increase in SBP group. Number of cases with shrunken liver was higher in SBP gp versus non-SBP gp, denoting that more deterioration of liver parynchyma is likely associated with SBP infection.

Our results showed that evaluated correlations between NLR and different parameters in each of the studied groups. In the SBP group, there was strong positive correlation for NLR with age of patient, WBCs count, neutrophils, lymphocytes, platelets, total and 
direct bilirubin, PT and INR, and negative correlation with albumin, Cr, HB, ALT and AST.

Our results showed that a multivariate analysis for the factors affecting SBP is illustrated. There was significant correlation between SBP infection and NLR, bleeding, bilhariziasis, WBCs count and history of drugs.Accordingly, it is plausible to assume that these factors are detrimental in the prognosis of patients with chronic liver disease and ascites.

Using ROC curve, it was shown that NLR level can be used to discriminate between patients and controls at a cutoff level of > 3.5, with $100 \%$ sensitivity, $42.86 \%$ specificity, $63.6 \%$ PPV and 100\% NPV. CRP level also can be used to discriminate between patients and controls at a cutoff level of > 43.3, with $77.14 \%$ sensitivity, $62.86 \%$ specificity, $62.86 \%$ PPV and $73.3 \%$ NPV. These findings agree with [9].

NLR can also be used as a diagnostic surrogate marker for SBP. This has previously been suggested by Lee et al [25]. In fact, combined NLR and CRP can be used as a novel noninvasive test for SBP diagnosis.

This study confirmed that NLR and CRP [which are almost routine laboratory tests] could be used as a novel, simple, low cost, noninvasive test for SBP diagnosis.

There are limitations to the current study that merit consideration. First, we included all patients with ascites, irrespective of the etiology. Second, the sample size was relatively small and larger studies are needed to evaluate this test in different clinical settings and to establish a reliable cutoff for CRP.

\section{References}

[1] P. Gines, E. Quintero, V. Arroyo. Compensated cirrhosis: natural history and prognostic factors. Hepatology.Vol. 7,PP.122-128,1997.

[2] G.Garcia-Tsao. Bacterial infections in cirrhosis: treatment and prophylaxis.Vol.J ,PP.40:749_756,2005.

[3] A.R. Bonnel, C.Bunchorntavakul, K.R.Reddy. Immune dysfunction and infection in patients with cirrhosisClinGastroentrolHepatol.Vol. 5,PP.9:727_738.s,2011.

[4] T.Reiberger, A.Ferlitsch, B.A.Payer. Non-selective betablocker therapy decreases intestinal permeability and serum levels of LBP and IL-6 in patients with cirrhosis.J Hepatol.Vol.58, PP.911921,2013.

[5] M.A Parsi, A.Atreja, ZeinNN. Spontaneous bacterial peritonitis: recent data on incidence and treatment.CleveClin J Med .Vol.71(7), PP.569$579,2004$.

[6] I.Pache, M.Bilodeau. Severe haemorrhage following abdominal paracentesis for ascites in patients with liver disease. Aliment Pharmacol Ther,Vol.21,PP.525-529,2005.

[7] K.Prystaz, K.Kaiser, A.Kovtun. Distinct Effects of IL-6 Classic and Trans-Signaling in Bone Fracture Healing. Am. J. Pathol,Vol. 188, PP.474-490 ,2018.
[8] XueTC, ZhangL, xieXY. prognostic significance of the neutrophil-to-lymphocyte ratio in primary liver cancer.a meta-analysis.PLOS ONE.Vol.9(5),PP.e96072,2014.

[9] A.Abdel-Razik, W.Eldars, RizkE. Platelet indices and inflammatory markers as diagnostic predictors for ascetic fluid infection.Vol.26,PP.1342$1347,2014$.

[10] R.Jalan, J.Fernandez, R.Wiest. Bacterial infections in cirrhosis:aposition statement based on the EASL Special Conference.Vol.60,PP.1310-1324,2014.

[11] M.Papp, Z.Vitalis, I.Altorjay. Acute phase proteins in the diagnosis and prediction of cirrhosis associated bacterial infections. Liver International,Vol.32(4), PP. 603-611,2012.

[12] N.Moreau, X.Wittebole, Y.Fleury. Neutrophil-tolymphocyte ratio predicts death in acute-on-chronic liver failure patients admitted to the intensive care unit: a retrospective cohort study. Shock [Augusta, Ga.],Vol.49(4), PP. 385,2018.

[13] N.Mousa, T.Besheer, A.Abdel-Razik. Can combined blood neutrophil to lymphocyte ratio and C-reactive protein be used for diagnosis of spontaneous bacterial peritonitis?. British journal of biomedical science.Vol.75(2), PP. 71-75, 2018.

[14] Y.J.Cai, J.J.Dong, J.Z.Dong. Neutrophillymphocyte ratio predicts hospital-acquired bacterial infections in decompensated cirrhosis. Clinica Chimica Acta,Vol.469, PP.201-207,2017.

[15] G.Pieri, B.Agarwal, A.K.Burroughs. C-reactive protein and bacterial infection in cirrhosis. Annals of Gastroenterology: Quarterly Publication of the Hellenic Society of Gastroenterology.Vol.27(2), PP.113, 2014.

[16] H.Summah, J.M.Qu..Biomarkers: a definite plus in pneumonia. Mediators Inflamm,Vol.4,PP. 675753:1-9,2009.

[17] M. Preto-Zamperlini, S.C.Farhat, M.B.Perondi. Elevated C-reactive protein and spontaneous bacterial peritonitis in children with chronic liver disease and ascites. J Pediatr Gastroenterol Nutr,Vol.8, PP. 58:96-9,2014.

[18] S.Liu, J.Ren, Q.Xia, X.Wu. Preliminary casecontrol study to evaluate diagnostic values of Creactive protein and erythrocyte sedimentation rate in differentiating active Crohn's disease from intestinal lymphoma, intestinal tuberculosis and Behcet's syndrome. Am J Med Sci,Vol. ,PP.346:467-472,2013.

[19] A.Tsiakalos, A.Karatzaferis, P.Ziakas. Acute phase protein as indicators of bacterial infection in patients with cirrhosis. Liver Int,Vol.15381542,2009..

[20] H.Wu, L.Chen, Y.Sun. The role of serum procalcitonin and C-reactive protein levels in predicting spontaneous bacterial peritonitis in patients with advanced liver cirrhosis. Pak J Med Sci. Nov-Dec.Vol.32(6),PP.1484-1488,2016.

[21] S.H.Janum, M.Søvs $\varnothing, \quad$ K.O.Gradel. C-reactive protein level as a predictor of mortality in liver 
disease patients with bacteremia. Scand J Gastroenterol.Vol.46(12),PP.1478-1483,2011.

[22] O.Le Moine, J.Deviere, J.M.Devaster. Interleukin6: an early marker of bacterial infection in decompensated cirrhosis. J Hepatol,Vol.20, PP.819-824,1994.

[23] J.P.Cervoni, T.Thevenot, D.WeilC-reactive protein predicts short-term mortality in patients with cirrhosis. J Hepatol,Vol.6, PP.1299-1304, 2012.
[24] B.Yildirim, R.Sari, N.IsciPatients with spontaneous bacterial peritonitis, and malignant and cirrhotic ascites. J Natl Med Assoc,Vol. 97, PP.276-280, 2005.

[25] J.H.Lee, S.Song, S.Y.Yoon. Neutrophil to lymphocyte ratio and platelet to lymphocyte ratio as diagnostic markers for pneumonia severity. Br J Biomed Sci. Jul.Vol.73(3),PP.140-142,2016. 